\title{
Combined selective descriptive medium for the culture of Neisseria gonorrhoeae
}

\author{
D E MACFARLANE \\ From the City Laboratory, Montrose Street, Glasgow
}

SUMMARY A selective identification medium for Neisseria gonorrhoeae was devised by incorporating antibiotics and a mixture of sugars into a yeast extract agar. The results were compared with those using a standard combined selective/non-selective blood agar medium. Recovery rates

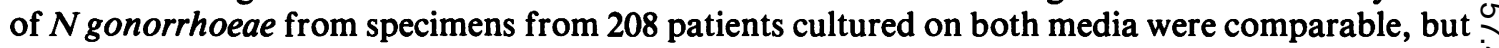
the selective descriptive medium identified 68 cultures of $N$ gonorrhoeae correctly, 64 of them after 24 hours' incubation.

\section{Introduction}

Laboratory confirmation of a diagnosis of gonococcal infection is usually considered essential and, in most instances, is achieved by cultural methods. A provisional diagnosis depends on finding typical intracellular clusters of Gram-negative diplococci in stained smears of secretions, and treatment is often based on a positive smear result. In men with acute gonococcal urethritis the stained smear is almost as accurate as proficient cultural methods ${ }^{1}$; in women with gonorrhoea about two-thirds of the confirmed cases can be diagnosed by a Gram-stained film. Thus, although a large number of patients will have been correctly diagnosed and treated before culture results are available, there are a significant number of gonococcal infections which can be diagnosed only by isolation and identification of the organism. Culture results are also important in cases giving a positive smear result, as they provide confirmation of the diagnosis, information on antibiotic sensitivities, and a test-of-cure after treatment.

Neisseria gonorrhoeae is usually identified from clinical specimens by isolation on a selective medium followed by speciation by fermentation reactions, which can take from two to seven days. A reduction in the time taken to obtain culture and antibiotic sensitivity results, and a simplification of the procedures, would obviously benefit both patients and staff of sexually transmitted disease (STD) clinics. A combined selective descriptive medium for the culture of $N$ gonorrhoeae has been devised and tested in this laboratory and at one of the local STD clinics.

Address for reprints: Dr D E Macfarlane, City Laboratory, 23 Montrose Street, Glasgow G1 1RN

Accepted for publication 31 December 1980

\section{Material and methods}

MEDIA

The selective medium, designated $\mathrm{G} 77 \mathrm{~S}$, was $\stackrel{5}{\stackrel{5}{+}}$ prepared according to the following formulation: $\vec{\theta}$ proteose peptone No 3 (Difco) $15.0 \mathrm{~g} ; \mathrm{K}_{2} \mathrm{HPO}_{4} \stackrel{\infty}{-}$ (Analar) $4.0 \mathrm{~g} ; \mathrm{KH}_{2} \mathrm{PO}_{4}$ (Analar) $1.0 \mathrm{~g} ; \mathrm{NaCl}$. (Analar) $5.0 \mathrm{~g}$; soluble starch (BDH) $1.0 \mathrm{~g}$; $\mathrm{Na}$ 六 $\mathrm{HCO}_{3}$ (Analar) $1.0 \mathrm{~g}$; $\mathrm{Fe}\left(\mathrm{NO}_{3}\right)_{3}$ (Analar) $2.0 \mathrm{mg}$ (as anhydrous salt); phenol red $(0.2 \%) 12 \mathrm{ml}$; Bacto agar $10.0 \mathrm{~g}$; and distilled water $935 \mathrm{ml}$. The medium $\mathbb{D}$ was autoclaved at $115^{\circ} \mathrm{C}$ for 15 minutes in sealed $\stackrel{\varrho}{\rightleftharpoons}$ containers to prevent loss of $\mathrm{CO}_{2}$ and then cooled to $45^{\circ} \mathrm{C}$ before the addition of $20 \mathrm{ml}$ of filter-sterilised $25 \%$ yeast extract solution, trimethoprim $2 \mathrm{mg}$, vancomycin $2.5 \mathrm{mg}$, polymixin B $5 \mathrm{mg}$, and nystatin 12000 units. Some batches of media were supplemented with $30 \mathrm{ml}$ of filter-sterilised $50 \%$ glucose solution $(\mathrm{G} 77 \mathrm{~S} / \mathrm{G})$ and others received $30 \mathrm{ml}$ of filter-sterilised $50 \%$ solutions of lactose, maltose, $\delta$ and sucrose (G77S/LMS), with a corresponding reduction of $60 \mathrm{ml}$ in the volume of distilled water. 음 The $\mathrm{pH}$ of the medium was adjusted to 7.4 with 7 $\mathrm{NaOH}$.

Blood agar was prepared in the laboratory from Oxoid Columbia agar base according to the manufacturers' instructions and supplemented with $\tilde{\circ}$ $5 \%$ horse blood. Selective blood agar was prepared $\tilde{\omega}$ by the further addition of the same antibiotic mixture ${ }^{\sigma}$ used in the preparation of the G77S medium. Bloodo agar and selective blood agar were combined in the $\mathbb{\Phi}$ two sections of a bi-plate (Sterilin) to give the? standard culture medium (SCM). A selective identifi- $\square$ cation (ID) system for $N$ gonorrhoeae was prepared $\stackrel{\vec{\Phi}}{\vec{D}}$ in three-compartment Petri-plates (Sterilin). One $\stackrel{\mathbb{\Omega}}{\stackrel{\Omega}{\mathscr{Q}}}$ section was filled with $G 77 \mathrm{~S} / \mathrm{G}$, one with $\stackrel{\complement}{\mathscr{Q}}$ G77S/LMS, and the third with blood agar. These IDo 
plates were compared with the SCM plates for the recovery and identification of $N$ gonorrhoeae from clinical material. The fermentation reactions of isolates on both media were confirmed on serum agar slopes consisting of Columbia agar base with 3\% rabbit serum, $1 \%$ carbohydrate, and a phenol red indicator.

\section{SPECIMEN COLLECTION AND CULTURAL TECHNIQUES}

Culture plates were prepared in the laboratory and transported to the clinics where they were stored in sealed containers at $4^{\circ} \mathrm{C}$. On the morning of a clinic session a number of plates were removed from the refrigerator and pre-warmed at $37^{\circ} \mathrm{C}$ before use. Specimens were taken with charcoal-coated swabs from the urethra and cervix of female patients, from the urethra of male patients, and from the oropharynx and rectum of both groups when indicated. The swabs were inoculated directly on to the three sections of the ID plates and on to the two sections of the SCM plates in a random order before incubation at $37^{\circ} \mathrm{C}$ in a moist candle jar. After overnight incubation at the clinic the candle jars containing the inoculated plates were transported to the laboratory, a short distance away, and examined on arrival and again after a further 24 hours' incubation at $37^{\circ} \mathrm{C}$. The ID plates were examined for the presence of oxidase-positive colonies of Gramnegative diplococci, which produced acid on the G77S/G medium but not on the G77S/LMS medium. These organisms were provisionally identified as $N$ gonorrhoeae, and sugar fermentation reactions were confirmed with serum agar slopes. The SCM bi-plates were also examined for the presence of oxidase-positive Gram-negative diplococci, and the fermentation patterns of these organisms were determined with serum agar slopes.

\section{Results}

The results of 208 cultures from different patients examined in parallel on both sets of media are shown in the table. $N$ gonorrhoeae was provisionally identified on 65 ID plates after 24 hours' incubation and on 68 plates after 48 hours' incubation. All oxidase-positive Gram-negative diplococci which gave a positive fermentation reaction on the G77S/G medium and a negative reaction on the G77S/LMS medium were confirmed as $N$ gonorrhoeae by their fermentation reactions on serum agar slopes. An additional four cultures of oxidase-positive Gramnegative diplococci were recovered from the ID plates. Two of these gave positive fermentation reactions on the G77S/G and G77S/LMS media and were subsequently identified as Neisseria
TABLE Comparison of a selective descriptive medium with a standard medium for the culture of Neisseria gonorrhoeae from clinical material

\begin{tabular}{|c|c|c|c|c|c|c|}
\hline \multirow{3}{*}{$\begin{array}{l}\text { Culture } \\
\text { medium }\end{array}$} & \multicolumn{4}{|c|}{ No of cultures: } & \multicolumn{2}{|c|}{ No of cultures positive on: } \\
\hline & \multicolumn{2}{|c|}{ Positive at } & \multirow[b]{2}{*}{ Negative } & \multirow[b]{2}{*}{ Total } & \multirow{2}{*}{$\begin{array}{l}\text { ID medium } \\
\text { only }\end{array}$} & \multirow{2}{*}{$\begin{array}{l}S C M \text { medium } \\
\text { only }\end{array}$} \\
\hline & $24 \mathrm{~h}$ & $48 \mathrm{~h}$ & & & & \\
\hline $\begin{array}{l}\text { ID* } \\
\text { SCM† }\end{array}$ & $\begin{array}{l}64 \\
69\end{array}$ & $\begin{array}{l}68 \\
71\end{array}$ & $\begin{array}{l}140 \\
137\end{array}$ & $\begin{array}{l}208 \\
208\end{array}$ & 7 & 8 \\
\hline
\end{tabular}

* Selective descriptive medium

+Standard selective/non-selective medium

meningitidis. One culture, which was recovered from the blood agar section of the ID plate, was also identified as $N$ meningitidis. The fourth culture, which did not break down carbohydrates on the ID plate, was identified as Branhamella catarrhalis. Very few organisms other than Neisseria species were recovered from the selective sugar media on the ID plates, and any such growth was usually restricted to a few colonies. Seven specimens gave positive results on ID plates and negative results on SCM plates, and in a further six specimens $N$ gonorrhoeae grew only on the selective sugar medium of the ID plates and not on the blood agar section.

After 24 and $\mathbf{4 8}$ hours' incubation on SCM plates oxidase-positive Gram-negative diplococci were detected in 73 and 75 cultures respectively. Subsequent sugar fermentation tests on serum agar slopes confirmed that 71 of these organisms were $N$ gonorrhoeae, three $N$ meningitidis, and one Br catarrhalis. Eight cultures which gave positive results on SCM plates gave negative results on the ID plates and five gave positive results on the selective blood agar only.

\section{Discussion}

All 68 cultures provisionally identified as $N$ gonorrhoeae on the ID plates were confirmed using serum agar slopes, and $94 \%$ of these cultures were correctly identified after overnight incubation. Presumptive identification of $N$ gonorrhoeae by colonial morphology and oxidase reaction was easier on the transparent G77S medium than on the opaque blood agar. With some cultures it was difficult to locate well-isolated colonies on the ID plates to determine their fermentation reactions, but this problem would be eliminated by proper plating techniques. Fewer positive cultures were detected on the blood agar sections of the ID and SCM plates than on the selective agar sections, mainly because of overgrowth with Proteus or other coliforms.

The recovery of $N$ gonorrhoeae from seven specimens on the ID plates which failed to grow on 
the SCM plates and from eight specimens on the SCM plates which failed to grow on the ID plates was somewhat surprising (table). Sampling error could not have been wholly responsible for these discrepancies since some specimens which gave negative results on one medium yielded a heavy growth on the other. Careful investigation of the procedures at the clinic showed that several deviations from the agreed protocol had occurred because the clinic staff lacked experience in direct plating techniques. Plates were sometimes left for long intervals at room temperature or at $37^{\circ} \mathrm{C}$ before incubation so that the agar surface became dry. Plates which had been inoculated were sometimes left at room temperature for several hours instead of being incubated immediately at $37^{\circ} \mathrm{C}$ in a sealed candle jar. This has been shown to influence the recovery rate of gonococci. ${ }^{2}$ Incorrect techniques may thus have been responsible for some growth failures. If an even distribution of errors is assumed, with a similar effect on both media, it appears that

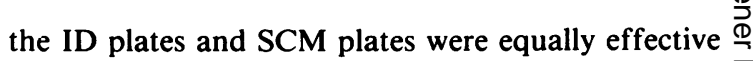
in recovering $N$ gonorrhoeae from clinical material, the $4 \%$ difference after 48 hours being within the limits of experimental error.

The addition of an antibiotic disc containing penicillin, ampicillin, or tetracycline to the blood agar section of the ID plate would provide additional rapid information on the antibiotic sensitivity of isolates. The zone sizes can be read more easily after the plate has been flooded with oxidase reagent.

\section{References}

1. McMillan A, Pattman RS. Evaluation of urethral culture for is Neisseria gonorrhoeae in the routine investigation of men attending a STD clinic. Br J Vener Dis 1979;55:271-3.

2. Chapel TA, Smeltzer M, Dassel R. The effect of delayed incubation in a $\mathrm{CO}_{2}$-enriched environment on gonococci. Health Lab Sci 1976; 13:45-8. 